\title{
Drug delivery systems for the photodynamic application of two photosensitizers belonging to the porphyrin family
}

\author{
Miryam Chiara Malacarne ${ }^{1,2} \cdot$ Stefano Banfi ${ }^{1}$ Matteo Rugiero ${ }^{1} \cdot$ Enrico Caruso $^{1}$ (i)
}

Received: 29 March 2021 / Accepted: 7 July 2021 / Published online: 14 July 2021

(c) The Author(s) 2021

\begin{abstract}
Photodynamic therapy involves the concomitant action of three components, light with an appropriate wavelength, molecular oxygen, and a molecule, able to absorb an electromagnetic radiation, called photosensitizer (PS). A fundamental aspect is the bioavailability of the PS that is directly related to some physicochemical properties of the PS itself as it should feature a certain degree of lipophilicity to easily cross the cell membrane, however, at the same time, should be sufficiently watersoluble to navigate in the bloodstream. Consequently, the use of a system for drug delivery becomes essential when photosensitizers with a high degree of lipophilicity are considered. In this work, we present three different drug delivery systems, microemulsions, emulsions and liposomes all capable of carrying a PS belonging to the porphyrin family: the tetraphenyl porphyrin (TPP) and the 4-hydroxyphenyl porphyrin (THPP), which show a relevant different degree of lipophilicity. A series of microemulsions (ME) and emulsions (E) were prepared, among which two formulations, one for THPP and one for TPP, have been chosen. The stability of these two carriers was monitored over time and under various temperature conditions. With the same criteria, two liposomal formulations have been also identified and analyzed. The four formulations mentioned above (one ME, one $\mathrm{E}$ and two liposomes) have been tested on SKOV3 tumor cell line comparing the photodynamic activity of the porphyrin formulations versus the aqueous/organic (DMSO) solution of the same two PSs. The results show that all the formulations have proved to be excellent carriers and that the liposomal formulation enhance the photodynamic efficacy of both porphyrins.
\end{abstract}

Keywords Microemulsion $\cdot$ Emulsion $\cdot$ Liposome $\cdot$ Photodynamic activity $\cdot$ Drug delivery

\section{Introduction}

The Photo-Dynamic chemo-Therapy (PDT) concerns the treatment of a localized pathology via the administration of a photosensitizer (PS), following irradiation of the diseased area with low-energy electromagnetic field (i.e. visible light) [1]. In clinical PDT, the patient is first treated with a solution of PS which preferentially accumulates in the tumor tissues because of their large vascularization. After a necessary incubation time, the cancer area is selectively irradiated with a light source emitting a low-energy visible radiation (with

Enrico Caruso

enrico.caruso@uninsubria.it

1 Department of Biotechnology and Life Sciences (DBSV), University of Insubria, Via J.H. Dunant 3, 21100 Varese, VA, Italy

2 PhD Student of the "Life Science and Biotechnology", University of Insubria, Varese, Italy $\lambda>650 \mathrm{~nm}$ ), thus resulting in the PS excited state. The loss of energy from the PS excited state can be achieved through two processes, called type I and type II, both requiring the interaction of the PS with its surroundings [2]. The type I process foresees that a PS, in its triplet-excited state, transfers the energy to the closest biomolecules generating free radicals reacting with oxygen, thus producing reactive oxygen species (ROS) [3, 4]. In the type II process, the PS transfers its energy directly to the surrounding molecular oxygen that attains the state of singlet oxygen, which is indeed a highly cytotoxic species. Although the two processes occur at the same time, the generation of singlet oxygen can be assumed as the predominant mechanism [5].

Many applications of photodynamic therapy are known, most of them are closely related to the methods of photosensitizer administration [6-9]. To this concern, the assessment of the characteristics of the drug is essential to predict the bioavailability of the drug itself. On the one hand, hydrophobicity could be a desirable feature, as the solubilization 
of the PSs in the lipid bilayer of the cell membrane has been pointed out to be one of the main factors determining PSs efficacy. On the other hand, this characteristic leads to the loss of PSs solubility in aqueous medium because of selfaggregation $[10,11]$. Furthermore, hydrophobicity complicates preparation of pharmaceutical formulations for parenteral administration [12]. However, this drawback could be avoided by formulating PSs into suitable carrier systems capable of transporting PSs in their stable, monomeric form, without altering their spectroscopic and functional properties. To be therapeutically useful, a PS formulation should deliver the PS in a form that should be readily and selectively internalized by the hyper proliferative cancer cells, in a way that facilitates accurate and convenient dosage. At the same time, the vector must be biodegradable and non-toxic as far it concerns its degradation products.

Different approaches have been developed to overcome the current shortcomings regarding the issues associated with the PS delivery, often resolved employing a delivery vehicle that enables a stable dispersion of PS in aqueous environment. To date, the most used vehicles are emulsions, microemulsions, liposomes and nanoparticles [13]. Emulsions are dispersions made up of two immiscible liquid phases, mixed together using mechanical stirring in the presence of a surfactant. Amphiphilic surface-active molecules are called surfactants and are responsible to reduce naturally existing attractive forces in the form of surface tension [14]. The choice of surfactants, based on hydrophilic-lipophilic balance (HLB) values, helps to develop the desired emulsion [15]. Micro-emulsion are clear, thermodynamically stable, isotropic liquid mixtures. They are prepared using oil, water, surfactant, and a co-surfactant and are made of very small particles, up to nano size, as compared to conventional emulsions [16]. Liposomes are one of the first nanoparticle-based delivery platforms to be applied in medicine [17], with over 11 liposomal formulations approved for clinical use today and many more in preclinical trials. They are concentric phospholipid vesicles consisting of single or multiple bi-layered membrane composed of natural or synthetic lipids [18]. Their unique ability to contain hydrophilic drugs in their aqueous core and hydrophobic agents within their lamellae, makes liposomes excellent carriers for therapeutic applications [19]. Tetraphenyl porphyrin (TPP) is highly lipophilic and poorly soluble in those solvents suitable for the in vitro cell treatments; therefore, it is necessary to find a carrier allowing the evaluation of TPP as potential photosensitizing agent; in the past, some systems have been presented to solve the problems related to its administration including biodegradable NPs based on poly(D, L-lactic acid), [20] pegylated NPs [21] or liposomal nano-formulations obtained by means of extrusion method [22]. On the contrary, tetra 4-hydroxyphenyl porphyrin (THPP), while remaining overall hydrophobic, featuring one hydroxy groups on each phenyl ring, is more hydrophilic than TPP and in fact in in vitro studies it can be easily formulated in a $\mathrm{H}_{2} \mathrm{O} / \mathrm{DMSO}$ mixture. Unfortunately, although DMSO may be present in low percentage $(<1 \%)$ in the stock solution for in vitro tests, it cannot be used for in vivo applications, consequently several methods of administration have been developed over the years including the glycoconjugation of porphyrin [23], encapsulation it in sterically stabilized liposomes [24] or complexation of THPP with cyclodextrins, as recently reported by many authors [25].

In this work we propose a series of drug delivery systems (microemulsions, emulsions and liposomes) for the photodynamic application of these two photosensitizers; the systems obtained will be completely characterized and the photodynamic efficacy compared with each other and in the case of THPP a comparison will also be made with the DMSO/ DMEM mixture.

\section{Experimental section}

\subsection{Chemical}

\subsubsection{General}

The equipment used to prepare emulsions, microemulsions and liposomes includes: MR 3001 magnetic heating plate; Laborota 4002 digital rotavapor (Heidolph); Sonorex RK156 ultrasonic bath (Bandelin); Mini Spray Dryer 190 (Büchi); Maximator® High Pressure Extruder HPE 12.0-100 homogenizer (CPLSACHSE); vortex Gene2 (Scientific Industries).

All chemicals reported in Table 1SI are commercial products. The aqueous phases were MilliQ deionized water or $1 X$ PBS buffer. The photosensitizers 5,10,15,20-tetraphenyl-21H,23H-porphyrin (TPP) and 5,10,15,20-tetra(4-hydroxyphenyl)-21H,23H-porphyrin (THPP) were commercial products (Sigma-Aldrich) and used as received (Fig. 1).

E, ME, and liposomes analyses have required the use of: Centrifuge 5702 and MiniSpin ${ }^{\circledR}$ plus centrifuge (Eppendorf); 713 pH Meter (Metrohm); Brookfield DV-E viscometer (TEM); Nanophox (Sympatec); Primo Star optical microscope (Carl Zeiss); Panda Niro Soavi homogenizer (GEA); UV-Probe 2550 spectrophotometer (Shimadzu).

\subsubsection{Microemulsion preparation}

The oil/water or water/oil microemulsions were prepared by mixing the dispersed phase in the dispersing one, with proper stirring. The conditions concerning the stirring intensity and the emulsification temperature were assessed case by case, according to manufacturer's specifications. Surfactants and co-surfactants were mixed in the dispersed or 
Fig. 1 Structure, chemical composition, and molecular weight of the two photosensitizers used

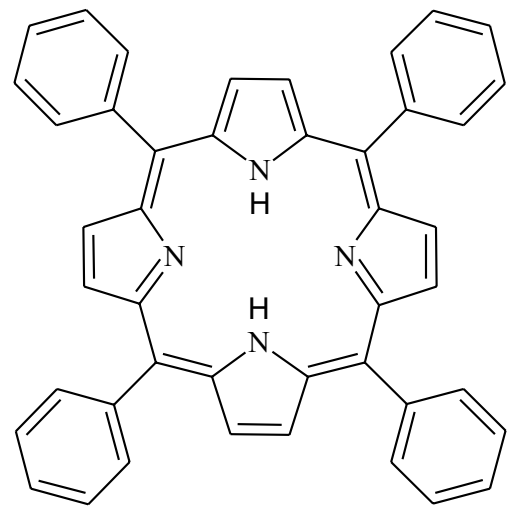

TPP

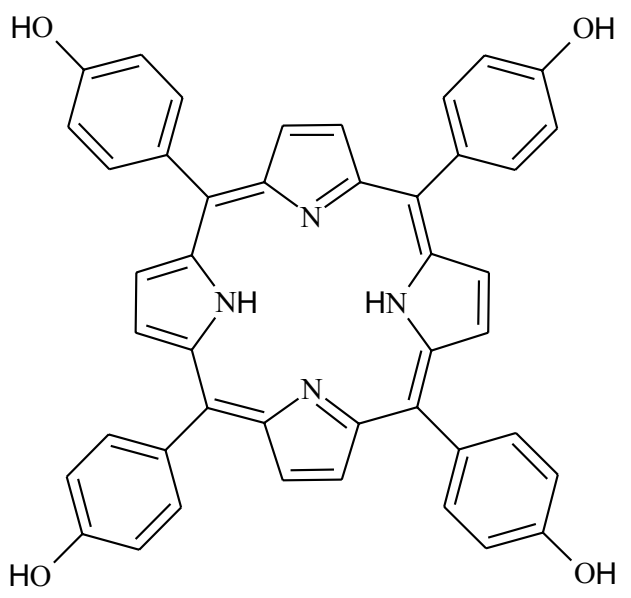

THPP
MW. $614.74 \mathrm{~g} / \mathrm{mol}$

$\mathrm{C}_{44} \mathrm{H}_{30} \mathrm{~N}_{4}$

MW. $678.73 \mathrm{~g} / \mathrm{mol}$

$\mathrm{C}_{44} \mathrm{H}_{30} \mathrm{~N}_{4} \mathrm{O}_{4}$

dispersing phase according to the physico-chemical characteristics (HLB, viscosity). Emulsification was carried out with both phases at the same temperature, dripping the dispersed phase into the dispersing one and keeping the emulsion under stirring for $10 \mathrm{~min}$, in the case of emulsification at RT, or allowing it to cool down until $25^{\circ} \mathrm{C}$ in case of high temperature emulsification (operating temperature $40-80{ }^{\circ} \mathrm{C}$ ).

\subsubsection{Emulsions prepared by high-pressure homogenization}

Emulsions prepared by high-pressure homogenization provide a first standard emulsification step, with a magnetic stirring heating plate, followed by the high-pressure homogenization step with Panda homogenizer from Niro Soavi Spa.

Surfactants were mixed in the oily phase and the mixture brought to a temperature of $70{ }^{\circ} \mathrm{C}$. The oily phase was then gradually added into the aqueous phase, also kept at $70^{\circ} \mathrm{C}$, under vigorous stirring (1000 rpm). The emulsion was then spilled into the homogenizer at a pressure between 800 and 1000 bar.

\subsubsection{Liposomes obtained with rotary evaporator and high-pressure filtration}

All the components which will form liposomes, with the only exception of the PS, are weighed (300 mg total) and dissolved in an appropriate solvent; if necessary, the mixture is sonicated to obtain a perfectly clear solution. This solution is then filtered by means of a syringe filters (porosity $0.45 \mu \mathrm{m}$ ), placed on a rotary evaporator equipped with a preheated bath, and allowed to rotate at $175 \mathrm{rpm}$ until the solvent evaporates completely. The film thus obtained was left under rotation for another $30 \mathrm{~min}$ at $60{ }^{\circ} \mathrm{C}$ and then definitively dried under a nitrogen flow for $5 \mathrm{~min}$. The dried lipid film was then hydrated by adding water until the film was completely dissolved and a dense and homogeneous solution was obtained (the use of vortex mixer is sometimes necessary). The procedure up to now described, allows obtaining "white" liposomes, i.e. not containing PS. To obtain the PS containing liposomes, $2 \mathrm{mg}$ of PS have been added to the homogeneous liposome solution at the end of the syringe filtration step. The liposomes were observed under a microscope to check the uniformity of the preparation and to confirm the absence of agglomerates. The analysis was carried out using a $100 \times$ and $1000 \times$ objective. The liposomal solution was then subjected to high-pressure filtration with Maximator HPE 12.0-100 homogenizer, using filters with a porosity range from 0.4 to $0.1 \mu \mathrm{m}$, and a pressure between 10 and 20 bar. This tool allows obtaining uniform liposomal suspensions with dimensions proportional to the porosity of the filter used. The final suspension was immediately collected in sterile falcons.

\subsubsection{Liposomes obtained with spray dryer}

All lipophilic components were dissolved in ethanol while the hydrophilic ones were dissolved in water; the alcoholic solution and the aqueous phase were then mixed and kept under stirring. In case of need, it was possible to proceed with heating and sonication steps to increase solubilization. When Büchi Mini Spray Dryer 190 has reached the operating temperature, the solution was loaded into the instrument and the atomization process was started (pumping speed $2 \mathrm{~mL} \mathrm{~min}^{-1}$; flow $700 \mathrm{Nl} \mathrm{h}^{-1}$ ). At the end of the process, the final product was collected in sterile falcons and kept under inert atmosphere. 


\subsection{Analyses}

\subsubsection{Particle size}

The measurements of the micelle particle sizes, which are present in both emulsions and microemulsions, were carried out by Photon Cross Correlation Spectroscopy (PCCS) using a Nanophox Sympatec instrument. This instrument allows determining the size and the stability of opaque suspensions or emulsions of nanoparticles in the range between 1 and $1000 \mathrm{~nm}$. Data will be re-elaborated in a Gaussian curve showing the average value of the micellar sizes and their distribution. Similarly, the particle sizes were also determined for filtered liposomes. In this case, liposomal solutions have been properly diluted and sonicated for 2 min before analysis, to remove any air bubbles.

\subsubsection{Microscopy}

Microscope analysis allows the detection of large particles $(>1 \mu \mathrm{m})$ and/or crystals. Microemulsions and emulsions were checked with a $1000 \mathrm{X}$ oil immersion lens using a Primo Star Carl Zeiss optical microscope equipped with an AxioCam Digital Camera. After the last filtration step, liposomal preparations were analyzed under a microscope with a $1000 X$ lens. The liposomes prepared following spray dryer procedure, were also analyzed to confirm the absence of any agglomerates after resuspension of the particulate.

\subsubsection{Centrifugation}

At the end of the preparation, each formulation was centrifuged with Eppendorf Mini Spin plus centrifuges or Eppendorf Centrifuge 5702, at $4000 \mathrm{rpm}$ for $10 \mathrm{~min}$, to evaluate a possible phase separation or the presence of undissolved material.

Liposomes were, instead, subjected to two different analyzes. In the first one, $500 \mu \mathrm{L}$ of "white" liposomes were centrifuged at $4000 \mathrm{rpm}$ over $4 \mathrm{~min}$ to verify the absence of phase separations. The second has been a gradient centrifugation $(500 \mu \mathrm{L}$ of $50 \%$ sucrose solution, $20 \mu \mathrm{L}$ of "white" liposomes and $500 \mu \mathrm{L}$ of milliQ water) at $4000 \mathrm{rpm}$ for 4 min to verify the homogeneity of the preparation.

\subsubsection{Determination of $\mathrm{pH}$}

The $\mathrm{pH}$ of all the formulations was measured using a 713 $\mathrm{pH}$ Meter Metrohm. The expected values should fall in the 6-7 pH unit range.

\subsubsection{Spectrophotometric analysis}

The absorbance of emulsions and microemulsions containing PS were spectrophotometrically determined with a Shimadzu UV-Probe 2550 spectrophotometer, using the corresponding molecule-free emulsion as blank.

For each liposomal preparation containing a PS, its final concentration was evaluated by measuring the absorbance at the wavelength of maximum absorption $(\lambda: 418 \mathrm{~nm})$. For each PS, a calibration line was prepared in the suitable solvent, DCM for TPP and methanol for THPP, respectively. In the case of THPP, an aliquot of the liposomal formulation was re-suspended in methanol and the absorbance read, after appropriate dilutions. A solution corresponding to the same liposomal preparation without PS and diluted in the same way, was used as blank. For TPP $2 \mathrm{~mL}$ of liposomal suspension were extracted with DCM. The organic phase was further diluted and analyzed spectrophotometrically at the appropriate wavelength. The same procedure was also applied to the "white" samples.

\subsubsection{Analyses of the stability}

The stability degree of different formulations was carried out by placing the solutions in vials, under inert atmosphere and in the dark, inside a stability box thermostatically controlled at three temperatures $\left(4,25\right.$, and $\left.40{ }^{\circ} \mathrm{C}\right)$. The stability of samples exposed to the light was carried out at room temperature. At predetermined times $(t: 0,7,15,30$, and 60 days), the samples were analyzed to evaluate different parameters, such as particle size, $\mathrm{pH}$ and absorbance; furthermore, the same samples were subjected to microscopic analysis to evaluate the appearance of crystals due to aggregation phenomena. A comparison was made with the stability of the corresponding "white" solutions.

\subsubsection{Viscosity}

The viscosity of emulsions and microemulsions was measured by means of a Brookfield DV-E Viscosimeter using the Brookfield RV Spindle 06, at $1 \mathrm{rpm}$.

\subsubsection{Lipid oxidation}

The stability of liposomal preparations can be also evaluated determining the percentage of oxidized lipids over time [26]. The oxidation of phospholipid fatty acids, in the absence of specific oxidants, occurs preferentially on lipids containing conjugated carbon-carbon double bonds; in fact, the presence of unsaturated carbon bonds makes these substances more likely to undergo oxidative degradation. The percentage of oxidized lipids was spectrophotometrically evaluated on the "white" liposomal formulations, to avoid the 
interference of the PS absorbance. At certain times, $100 \mu \mathrm{L}$ of "white" liposomes was diluted in water to obtain a final phosphatidylcholine concentration of $10 \mathrm{mg} \mathrm{mL}^{-1}$. From this solution, $300 \mu \mathrm{L}$ was withdrawn and treated with $300 \mu \mathrm{L}$ of absolute ethanol; $100 \mu \mathrm{L}$ of this last solution was further diluted with $3 \mathrm{~mL}$ of ethanol. Finally, we proceeded with the reading of the absorbance at $233 \mathrm{~nm}$. The percentage of oxidized lipids was then calculated with the following equation:

$\%$ oxidation $=\frac{\mu \mathrm{mol} / \mathrm{mL} \text { diene }}{\mu \mathrm{mol} / \mathrm{mL} \text { phosphatidylcholine }} \times 100$.

The initial concentration of dienes was calculated measuring the absorbance at $233 \mathrm{~nm}$, using the Lambert-Beer law (molar extinction coefficient $\varepsilon=30,000$ ).

\subsection{Biological studies}

\subsubsection{General}

The human ovarian carcinoma cells SKOV3 were obtained from the American Type Culture Collection (Rockville, MD, USA) and maintained in DMEM/F-12 (Dulbecco's Modified Eagle Medium/Nutrient Mixture F-12, Sigma-Aldrich) supplemented with $10 \%$ fetal bovine serum, $1 \%$ glutamine and $0.5 \%$ antibiotic mixture (penicillin, streptomycin, and neomycin) (Sigma-Aldrich), in standard culture conditions at $37{ }^{\circ} \mathrm{C}$ in a humidified $5 \% \mathrm{CO}_{2}$ atmosphere.

Emulsions and microemulsions under study were previously filtered by syringe ( $0.2 \mu \mathrm{m}$ filter) to minimize the risk of contamination. On the contrary, liposomes $(0.5 \mathrm{~g})$ suitable for biological tests were just re-suspended in $5 \mathrm{~g}$ of sterile PBS buffer solution.

\subsubsection{Cytotoxic studies}

The anti-proliferative effect of each compound was assessed using the MTT assay [27]. Briefly, $1 \times 10^{4}$ cells $\mathrm{mL}^{-1}$ were seeded onto 96-well plates and allowed to grow for $48 \mathrm{~h}$ prior to the treatment with different PS concentrations. After $24 \mathrm{~h}$, the drug-containing medium was replaced by fresh PBS, and cells were irradiated under visible light for $2 \mathrm{~h}$ using a $500 \mathrm{~W}$ tungsten-halogen lamp, characterized by an irradiance of $22 \mathrm{~mW} \mathrm{~cm}{ }^{-2}$ (an average value determined between 380 and $780 \mathrm{~nm}$ with a Licor- 1800 spectroradiometer) and a fluence of $158.4 \mathrm{~J} \mathrm{~cm}^{-2}$. With the $500 \mathrm{~W}$ halogen lamp, a cooling apparatus is necessary to avoid cell death caused by over-heating; thus, a flowing water filter was placed between the light source and plate containing cells, thus maintaining a temperature of approximately $37^{\circ} \mathrm{C}$ below the filter. At the end of irradiation period, the cells were incubated in the dark at $37^{\circ} \mathrm{C}$ in drug-free medium and $24 \mathrm{~h}$ later MTT was added to each well (final concentration
$0.4 \mathrm{mg} \mathrm{mL}^{-1}$ ). The blue Formazan crystals, formed through MTT metabolism by viable cells, were dissolved in DMSO and optical densities were measured at $570 \mathrm{~nm}$ using a Universal Microplate Reader EL800 (Bio-Teck-Instruments). In control samples, the same procedure was used except for the treatment with the PS. $\mathrm{IC}_{50}$ values were estimated according to the concentration-response curves by non-linear regression analysis, using GraphPad Prism software, v. 5.0 (GraphPad, San Diego, CA, USA).

Possible intrinsic cytotoxic effects (i.e., not photo induced cell death) of the porphyrins were assessed on control cultures kept in the dark and treated as described above.

\subsubsection{Cellular uptake and intra-cellular localization}

To assess PSs uptake, SKOV3 cells were seeded onto 6-well plates $\left(2.5 \times 10^{5}\right.$ cells/well) and exposed to THPP (DMSO/ DMEM), ME1-THPP and L9-THPP (100 nM for $24 \mathrm{~h}$ ) solutions; in these experiments, cells were kept in the dark (not irradiated). At the end of the exposure time, the cells were detached by trypsinization, washed thoroughly in ice-cold PBS, resuspended in PBS and analyzed.

All samples were analyzed with a Becton Dickinson FACScalibur instrument equipped with a $15 \mathrm{~mW}, 488 \mathrm{~nm}$, air-cooled argon laser and data were analyzed using Cell QuestPRO software (Becton Dickinson). PSs uptake was quantitated in arbitrary units based on the mean fluorescence intensity (MFI), collecting fluorescein fluorescence through a $530 \mathrm{~nm}$ band-pass filter and PSs fluorescent emission through a $575 \mathrm{~nm}$ band-pass filter, respectively. For the cytometric analysis, the treatment with PSs was omitted in control samples [28]. The intra-cellular localization of THPP was carried out as previously reported [29], using all the above-mentioned solutions. Briefly, SKOV3 cells were seeded onto coverslips $(10,000$ cells), allowed to grow for $48 \mathrm{~h}$, and subsequently treated with a $5 \mu \mathrm{M}$ PS concentration. After $24 \mathrm{~h}$, cells attached to coverslips were washed three times in PBS, fixed in 3\% paraformaldehyde for at least $10 \mathrm{~min}$ and again washed in PBS three times. Coverslips were mounted on microscope slides and images were acquired using a LEICA TCS SP8 X confocal laser-scanning microscope.

\subsection{Statistical analysis}

The experiments were repeated at least four times on separate dates. $\mathrm{IC}_{50}$ values (i.e. the concentration affecting $50 \%$ of cell survival fraction) were obtained by nonlinear regression analysis, using the GraphPad PRISM 3.03 software (GraphPad Software Inc., San Diego CA). Data, normally distributed, were analyzed by means of one-way ANOVA (origin_7.0 SR0; Origin lab, Origin Lab Corporation 
Nothampton, Massachusetts, USA). Significant treatment effects were estimated $(p<0.05, p<0.01$ and $p<0.001)$.

\section{Results and discussion}

\subsection{Emulsions and microemulsions: solubility}

Some PS-free formulations ("white" formulations) were initially studied to understand which one of the compositions could be more stable, then, the chosen formulations were prepared with the addition of PS. To this purpose, the PS was dissolved in the oil phase or in the main surfactant, heating and stirring the phase of interest. To obtain the best lipophilic phase, solubility tests were carried out solubilizing the PS in various lipophilic vehicles. The best results are reported in supporting information Table 2SI.

\subsection{Microemulsions (ME): formulations, characterizations, and stability analysis}

According to the PS solubility results, oil-in-water (O/W) or water-in-oil (W/O) formulations were prepared, choosing suitable surfactants on the base of the physicochemical characteristics of the vehicle and that one of the PS (see supplementary information Table 3SI). Research has only focused on the use of lipophilic phases suitable in pharmaceutical applications.

Among the "white" ME prepared, only three of the O/W types (ME1, ME5 and ME12) (Table 1), showed clarity and absence of phase separations one hour after preparation, consequently they were re-prepared by dissolving $0.0025 \%$ by weight of PS in the oily phase. After the addition of the PS, ME5-PS and ME12-PS showed a phase separation, thus were excluded from further stability tests. On the contrary, ME1-PS proved to be stable and then was characterized evaluating $\mathrm{pH}$, viscosity, and the possible presence of crystals by microscopic analysis. Centrifuge tests were also used to confirm the stability of the formulation, evaluating the absence of phase separation and the absence of precipitate. Since ME1 is an O/W formulation, evaluation of the particle size at the Nanophox was not made possible, due to a difficult determination of the dispersant-phase density.

Table 1 Microemulsions O/W type, stable and clear one hour after preparation

\begin{tabular}{ll}
\hline O/W & Composition \\
\hline ME1 & $7 \%$ Labrasol $+1 \%$ Transcutol P $+92 \% \mathrm{H}_{2} \mathrm{O}$ \\
ME5 & $25 \%$ Labrasol $+5 \%$ Lauroglycol $90+10 \%$ \\
& Tween $80+50 \% \mathrm{H}_{2} \mathrm{O}$ \\
ME12 & $34.3 \%$ Labrasol $+5.7 \%$ Lauroglycol \\
& $90+30 \%$ Gelucile $44 / 11+30 \% \mathrm{H}_{2} \mathrm{O}$ \\
\hline
\end{tabular}

The formulations resulting stable in the preliminary analyses, were further studied under different conditions and times. Thus, these formulations were placed in the dark in a stability box kept at $4,25,40{ }^{\circ} \mathrm{C}$ and at RT in presence of light. All samples were subsequently analyzed at 7, 15, 30 and 60 days for the same parameters (Table 2).

From the data reported in Table 2, it can be observed that the viscosity remains unchanged in ME1-PS. Furthermore, the two ME1-PS stored at $4{ }^{\circ} \mathrm{C}$ showed no changes in $\mathrm{pH}$ over 60 days while, in the other conditions, a slight $\mathrm{pH}$ decrease was observed. Finally, ME1-THPP never showed the appearance of crystals while the ME1-TPP showed the presence of some crystals already after 7 days at all temperatures considered, both in the dark and in the light. These last data clearly indicate that this ME does not allow a permanent and stable solubility of the highly lipophilic TPP. For an indepth analysis of the stability of the ME-PS thus formulated, the absorbance variations over time were analyzed spectrophotometrically (see Supplementary information Fig. 1SI). For a more correct and exhaustive interpretation, the decrease in the absorbance was calculated and reported in percentage of the residual absorbance after 60 days (Fig. 2).

The absorbance data agree with the data reported in Table 2 concerning the stability of the two ME-PS; actually, the ME1-THPP showed excellent conservation at 4 and $25^{\circ} \mathrm{C}$, as only a partial decay of the signal relative to the PS being observed at $40{ }^{\circ} \mathrm{C}$ after 60 days. A higher degradation rate was measured for the sample kept in presence of light due to a phenomenon probably related to a partial photobleaching effect. These results confirm the high stability of ME1-THPP; therefore, this has been the microemulsion chosen for the subsequent biological tests.

The low stability of ME1-TPP was confirmed considering the decrease of the absorbance with time. In fact, although it is known that the stability of the ME is temperaturedependent, unfortunately, at the lowest considered temperature $\left(4^{\circ} \mathrm{C}\right)$ the absorbance of the microemulsion showed a $70 \%$ decrease after 60 days. The absorbance was null $(100 \%$ decrease) at $40{ }^{\circ} \mathrm{C}$ after the same period. In this case, the decrease or disappearance of the porphyrin absorbance signal could be related to the formation of insoluble aggregates. A similar result was, obviously, observed for sample stored at RT under the light, when the intrinsic instability is worsened by the photobleaching effect. Overall, it can be concluded that the choice of microemulsion formulations is not suitable for the TPP photodynamic application.

\subsection{Emulsions: formulations, characterizations, and stability analysis}

Due to the instability of ME1-TPP, it has been decided to prepare an emulsion using the high-pressure homogenization technique. In the first step, two oily phases were chosen, 
Table 2 Results of the stability analyses carried out on clear ME1

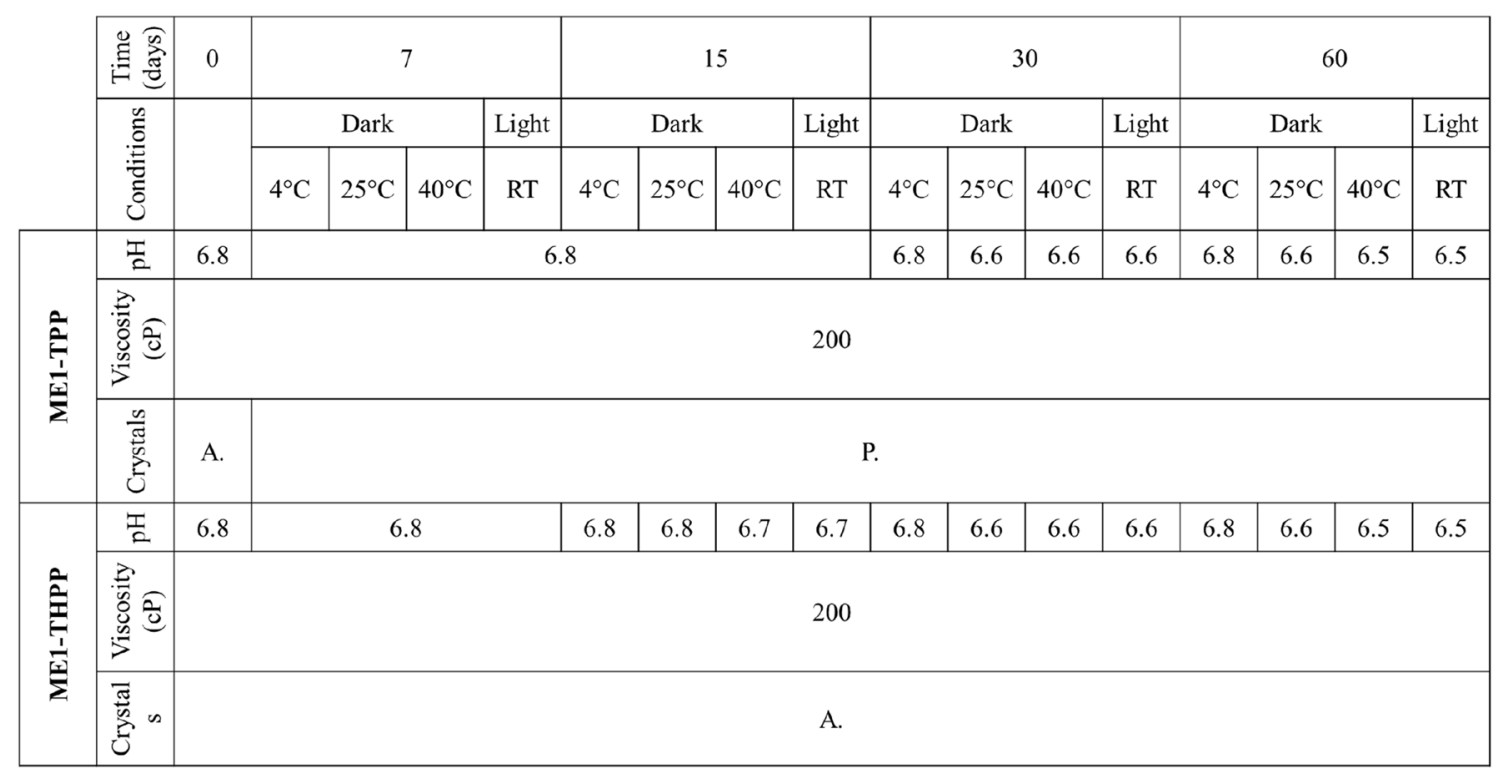

The $\mathrm{pH}$, the viscosity, and the presence of crystals in the formulation were determined at 7, 15, 30 and 60 days after preparation of the ME. The analyses were determined at $4,25,40{ }^{\circ} \mathrm{C}$ in the dark and at $\mathrm{RT}$ in the light. A stands for absence of crystals while P means the presence

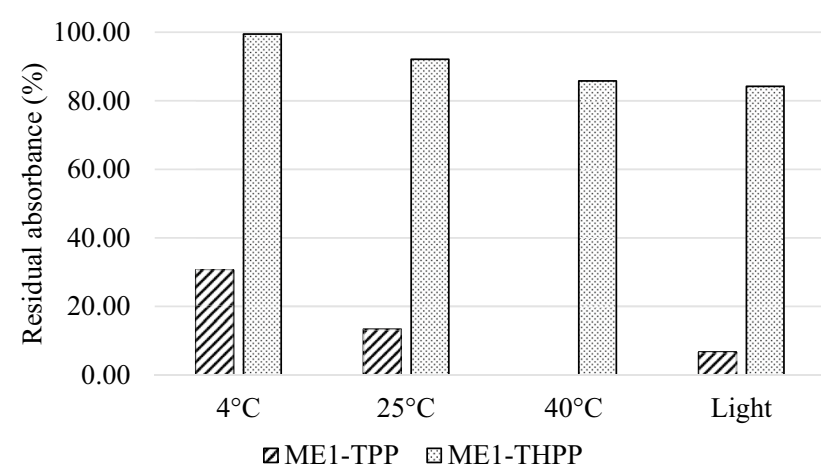

Fig. 2 Residual absorbance detected on ME1-PS after 60 days of analysis in the dark at $4,25,40{ }^{\circ} \mathrm{C}$ and under light at RT. The values are reported as percentage with respect to the initial value of absorbance $(t=0)$, corresponding to $100 \%$

based on the solubility (Table 2SI), then TPP was dissolved in Lauroglycol 90 (E1) and in Propylene Glycol Dipelargonate (DPPG) (E2). Furthermore, considering a possible topical application of the formulation for skin cancers, a third emulsion (E3) was prepared using DPPG in combination with almond oil, a compound known for its moisturizing properties and with reduced irritating effects on the skin. Preservatives and chelating agents have been added to these emulsions to increase their stability (Table 3).

As E3 was the only stable and uniform formulation $24 \mathrm{~h}$ after preparation, it was then re-prepared with the addition of TPP, dissolving $0.0025 \%$ by weight of the porphyrin in the oily phase. The same stability analyses made for ME were then repeated in the case of E3-TPP (Table 4).

The absorbance of this formulation was also measured at $418 \mathrm{~nm}$ with a spectrophotometer at fixed times (See Supplementary information Fig. 2SI) (Fig. 3).

The data reported in Table 4 and the residual absorbance after 60 days (Fig. 3) clearly indicate an interesting stability of the formulation. E3-TPP showed only a limited decrease in absorbance (more relevant in the case of the formulation exposed to light), absence of crystals, no variation in $\mathrm{pH}$ and viscosity (slight decrease in viscosity only for the emulsion

Table 3 The three emulsions prepared, and the relative formulation expressed in W/W \%

\section{Composition}

E1 $30 \%$ Lauroglycol $90+3 \%$ Transcutol P $+10 \%$ Propylen glycol $+2 \%$ Montanov $68+3 \%$ BrijS $20+1.2 \%$ Tween $80+1 \%$ Phenoxyethanol $+0.1 \%$ EDTA $+49.7 \% \mathrm{H}_{2} \mathrm{O}$

E2 $30 \%$ DPPG $+2.5 \%$ Transcutol P $+10 \%$ Propylen glycol $+2 \%$ Montanov $68+3 \%$ Brij S20 $+1.2 \%$ Tween $80+1 \%$ Phenoxyethanol $+0.1 \%$ $\mathrm{EDTA}+50.2 \% \mathrm{H}_{2} \mathrm{O}$

E3 15\% DPPG + 15\% Almond oil + 2\% Transcutol P+10\% Propylen glycol + 2\% Montanov 68 + 3\% Brij S20 + 1.2\% Tween $80+1 \%$ Phenoxyethanol $+0.1 \%$ EDTA $+50.7 \% \mathrm{H}_{2} \mathrm{O}$

Total weight of the emulsion $200 \mathrm{~g}$ 
Table 4 Results of the stability analyses carried out on clear E3-TPP

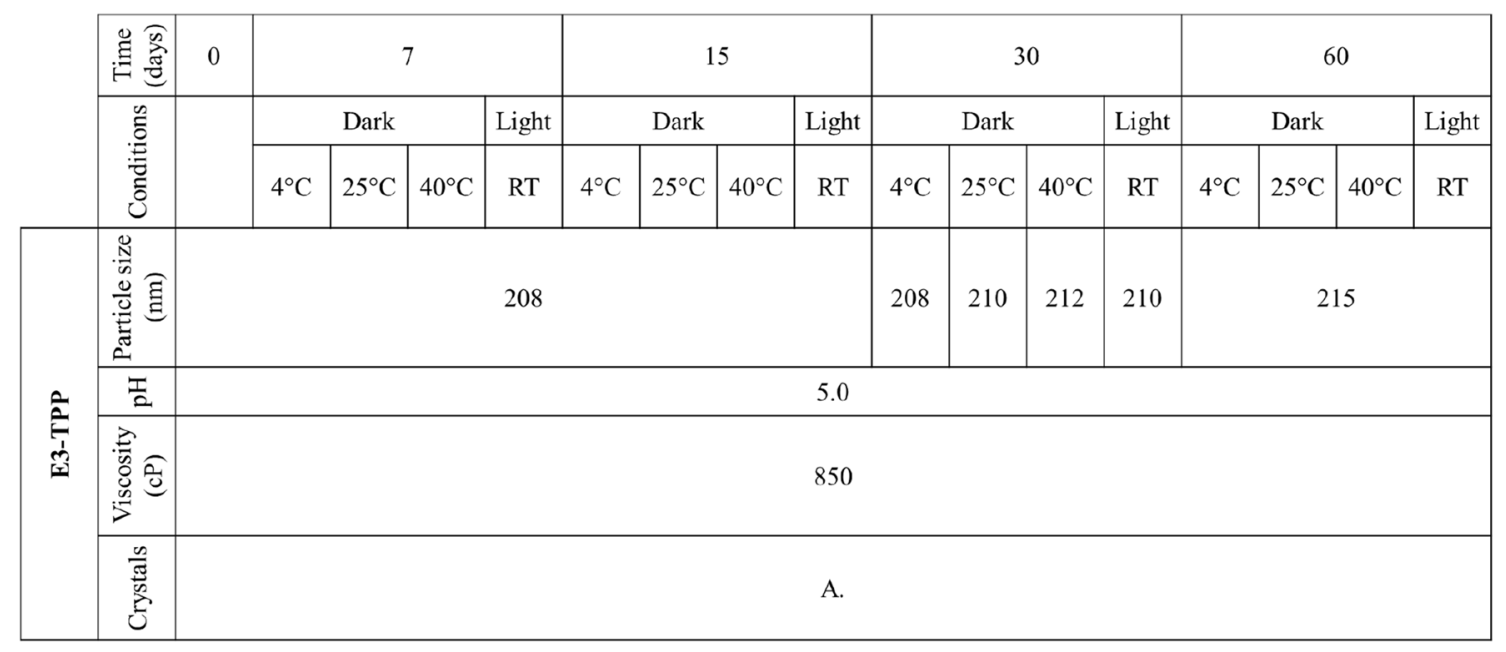

The particle size, the $\mathrm{pH}$, the viscosity, and the presence of crystals in the formulation were determined at $7,15,30$ and 60 days after preparation of the ME. The analyses were determined at $4,25,40{ }^{\circ} \mathrm{C}$ in the dark and at RT in the light. A stands for absence of crystals

100.00

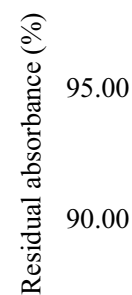

85.00

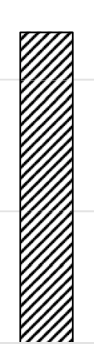

$4^{\circ} \mathrm{C}$

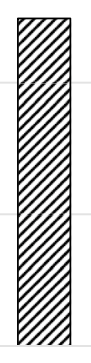

$25^{\circ} \mathrm{C}$

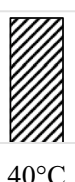

$40^{\circ} \mathrm{C}$

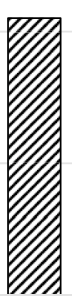

Light
Fig. 3 Residual absorbance detected on E3-TPP after 60 days of stability in dark at $4,25,40{ }^{\circ} \mathrm{C}$ and under light at RT. The values are reported as percentage with respect to an initial absorbance value, measured at $t=0$, corresponding to $100 \%$

kept at $40{ }^{\circ} \mathrm{C}$ ) and not relevant variations of the micellar size. Consequently, E3-TPP will be used for subsequent biological analyses.

\subsection{Liposomes: formulations, characterizations, and stability analysis}

Traditional method for liposomes preparation is the hydration of a film formed in a round bottomed flask following the evaporation of the low boiling organic solvent of a lipid mixture by means of a rotary evaporator [30].

The main problem with these preparations is the high degree of microbial contamination in the final product; this contamination can be avoided using sterile aqueous phases and filtering the solution with $0.2 \mu \mathrm{m}$ porosity syringe-filter.

In preliminary studies, various liposomal formulations with different excipients were prepared, evaluating their stability by means of microscopic analyses (see supplementary information Table 4SI and Fig. 3SI).

Formulations L3 (Lipoid SPC-3/Lipoid DSPG-Na $+7 \%$ Cholesterol $+0.4 \% \alpha$-tocopherol; solvent: methanol/DCM) was the most uniform as the use of the methanol/DCM mixture allowed the best solubilization of the components.

When THPP was added in the L3 formulation, a color change of the PS from purple to green was observed because of the slightly acidic pH of the liposome, an attempt was made changing the phospholipid thus obtaining two new variants (L8 and L9) (Table 5).

L9-THPP, containing Lipoid S100 as basic phospholipid, gave the best results thus the same liposome, in which TPP was dispersed, was also prepared (Table 5).

As can be seen from the data reported in Table 5, appropriate formulations were required for each PS, depending on the lipophilicity and structure of the molecule itself. Actually, different PSs intercalate differently in the lipid layer, and this determines the formation of stable or not-stable liposomes, depending on the interaction with the lipophilic components of the bilayer. In the case of TPP, the addition of fatty acid was necessary to stabilize the liposome.

To test the liposome stabilities, $1 \mathrm{~mL}$ of L9-THPP or L13TPP was diluted in $100 \mathrm{~mL}$ of $1 \mathrm{X}$ PBS buffer and filtered in two steps (first $0.45 \mu \mathrm{m}$ and then $0.2 \mu \mathrm{m}$ filter); the solution was spectrophotometrically analysed before and after filtration, detecting an overall loss of about $30 \%$ of PS. For this reason, we opted for high-pressure filtration with Maximator homogenizer, with two consecutive filtration steps using $0.45 \mu \mathrm{m}$ and $0.1 \mu \mathrm{m}$ filters, obtaining liposomes with negligible PS loss $(<0.5 \%)$ [31]. Liposomes thus obtained were characterized measuring the particle size and the $\mathrm{pH}$, followed by microscopic analyses and, finally, by centrifuge test at predetermined times (Table 6). To limit microbial 
Table 5 Composition of liposomal formulations for THPP and TPP, with comments on the characteristics of the preparation

\begin{tabular}{lll}
\hline & Composition & Comments \\
\hline L3-THPP & Lipoid SPC-3/Lipoid DSPG-Na $+0.4 \% \alpha$-tocopherol $+7 \%$ cholesterol & Dark green lipid film \\
L8-THPP & Lipoid S20/Lipoid DSPG-Na $+0.4 \% \alpha$-tocopherol +7\% cholesterol & Dark green lipid film \\
L9-THPP & Lipoid S100 + DSPG-Na $+0.4 \% \alpha$-tocopherol $+7 \%$ cholesterol & Homogeneous liposomes with purple color \\
L10-TPP & Lipoid S100+DSPG-Na $+7 \%$ cholesterol & Not encapsuled/precipitated porphyrin \\
L11-TPP & Lipoid S100 & Not encapsuled/precipitated porphyrin \\
L12-TPP & Lipoid S100 $+6 \%$ squalane $+6 \%$ Labrafac & Not encapsuled/precipitated porphyrin \\
L13-TPP & Lipoid S100+2.5\% myristic acid & Homogeneous liposomes with purple color \\
\hline
\end{tabular}

Table 6 Results of the stability analyses carried out on liposomes L9-THPP and L13-TPP

\begin{tabular}{|c|c|c|c|c|c|c|c|c|c|c|c|c|c|c|c|c|c|c|}
\hline & 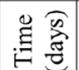 & 0 & \multicolumn{4}{|c|}{7} & \multicolumn{4}{|c|}{15} & \multicolumn{4}{|c|}{30} & \multicolumn{4}{|c|}{60} \\
\hline & \multirow{2}{*}{ 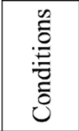 } & & \multicolumn{3}{|c|}{ Dark } & \multirow{2}{*}{\begin{tabular}{|c|} 
Light \\
RT \\
\end{tabular}} & \multicolumn{3}{|c|}{ Dark } & \multirow{2}{*}{\begin{tabular}{|c|} 
Light \\
RT \\
\end{tabular}} & \multicolumn{3}{|c|}{ Dark } & \multirow{2}{*}{\begin{tabular}{|c|} 
Light \\
RT
\end{tabular}} & \multicolumn{3}{|c|}{ Dark } & \multirow{2}{*}{\begin{tabular}{|c|} 
Light \\
RT
\end{tabular}} \\
\hline & & & $4^{\circ} \mathrm{C}$ & $25^{\circ} \mathrm{C}$ & $40^{\circ} \mathrm{C}$ & & $4^{\circ} \mathrm{C}$ & $25^{\circ} \mathrm{C}$ & $40^{\circ} \mathrm{C}$ & & $4^{\circ} \mathrm{C}$ & $25^{\circ} \mathrm{C}$ & $40^{\circ} \mathrm{C}$ & & $4^{\circ} \mathrm{C}$ & $25^{\circ} \mathrm{C}$ & $40^{\circ} \mathrm{C}$ & \\
\hline \multirow{3}{*}{ 育 } & 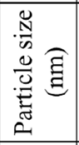 & 165 & 165 & 168 & 212 & 163 & 165 & 180 & - & 188 & 190 & 192 & - & 190 & 245 & 242 & - & 240 \\
\hline & $\frac{\pi}{2}$ & 6.5 & 6.5 & 6.5 & 6.4 & 6.4 & 6.4 & 6.4 & 6.2 & 6.2 & 6.4 & 6.0 & 6.0 & 6.0 & 6.1 & 6.0 & 5.8 & 6.0 \\
\hline & 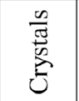 & \multicolumn{17}{|c|}{ A. } \\
\hline \multirow{3}{*}{ 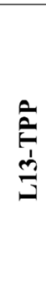 } & 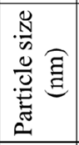 & 170 & 170 & 185 & 212 & 190 & 170 & 196 & 215 & 196 & 180 & 196 & 1 & 199 & 1 & 196 & 1 & 170 \\
\hline & $\frac{\pi}{2}$ & 6.5 & 6.5 & 6.5 & 6.5 & 6.5 & 6.5 & 6.4 & 6.4 & 6.5 & 6.5 & 6.3 & 6.1 & 6.3 & 6.3 & 6.0 & 6.5 & 6.0 \\
\hline & 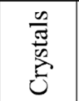 & \multicolumn{17}{|c|}{ A. } \\
\hline
\end{tabular}

The particle size, the $\mathrm{pH}$, and the presence of crystals in the formulation were determined at 7, 15, 30 and 60 days after preparation of the liposomes. The analyses were determined at $4,25,40{ }^{\circ} \mathrm{C}$ in the dark and at RT in the light. A stands for absence of crystals

contamination, $0.5 \%$ phenoxyethanol was added, as a preservative, to each stable formulation.

The overall data of the analyses concerning the stability of the two liposomal formulation, showed that the $\mathrm{pH}$ did not change in the various conditions tested and that no crystal formation was observed. On the other hand, a progressive increase in micellar size was observed, a phenomenon particularly relevant for the samples placed at $40{ }^{\circ} \mathrm{C}$, for which was no longer possible to carry out the analysis after a few days, probably because of the irregular dimensions assumed by liposomes.

An accurate assessment of the spectrophotometric stability was carried out here too, evaluating the change in the absorbance intensity for each formulation (see Supplementary information Fig. 4SI). The analyses were carried out at fixed days, covering an interval of 60 days. For a more correct and exhaustive interpretation of the data, the decrease in absorbance, with respect to time, of each formulation was measured and reported as the percentage of residual absorbance after 15 and 60 days of test in the dark at $4,25,40{ }^{\circ} \mathrm{C}$ and in the light at RT (Fig. 4).

Liposomal formulations, compared to ME, proved to be less stable about PS, under all conditions tested. None of the liposomal formulations was found stable after 60 days when kept at $40{ }^{\circ} \mathrm{C}$ or in the light, whereas it should be noted that both L13-TPP and L9-THPP maintains a good level of stability at 4 and $25^{\circ} \mathrm{C}$ for at least 7 days and L13-TPP seems to be quite stable also after 60 days at those temperatures ( $<80 \%$ at $4{ }^{\circ} \mathrm{C}$ and $70 \%$ at $25{ }^{\circ} \mathrm{C}$, respectively). On the contrary, L9-THPP already showed a strong loss of absorption when kept at $4{ }^{\circ} \mathrm{C}$ for 60 days. Both liposomal formulations undergo a strong photobleaching effect, which is such as to make them unusable after 7 days of storage under light.

For a correct and global interpretation of the data, the percentage of oxidized lipids was also analyzed (Fig. 5). The analysis was carried out at RT on "white" liposomes 


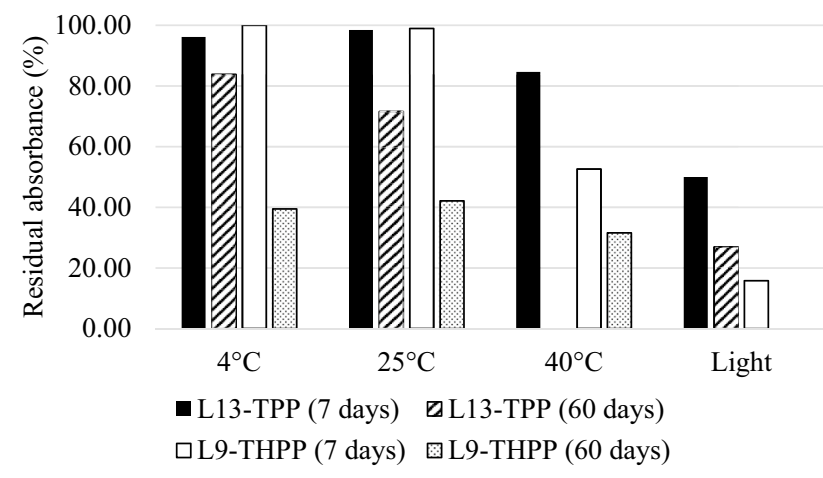

Fig. 4 Residual absorbance detected in Liposomes after 7 and 60 days of stability in dark at $4,25,40{ }^{\circ} \mathrm{C}$ and under light at RT. The values are reported as percentage with respect to the initial absorbance value $(t=0)$, corresponding to $100 \%$

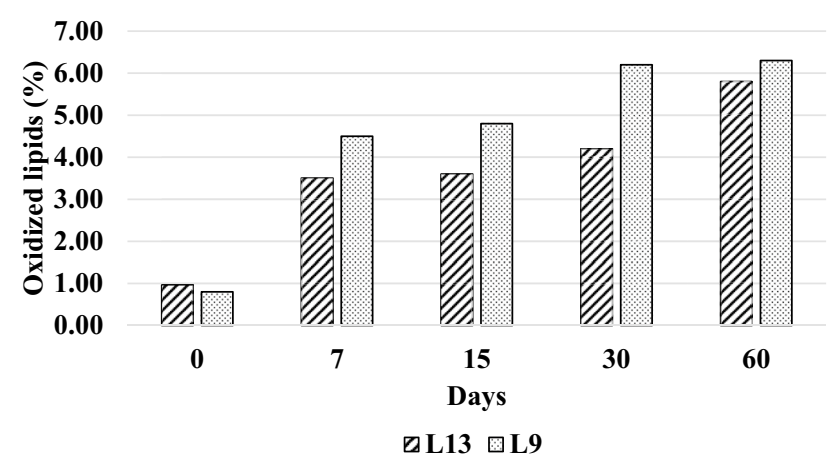

Fig. 5 Increase in percentage of oxidized lipids in the two liposomal formulations maintained at room temperature

corresponding to the above reported L-PS. The analysis could not be carried out on liposomes charged with the PS, due to the spectral interference of the PSs absorbance.

In general, the comparison of the analyses on oxidized lipids and the graph showing the spectrophotometric stability of the formulations at two different conservation times, does not allow establishing a direct correspondence. The instability of the formulations at long time (60 days) does not therefore depend on the spontaneous oxidation of lipids by molecular oxygen, however the concomitant presence of the PSs and light might be the cause of faster lipids oxidation.

By considering the whole data obtained on the stabilities of the lipid formulations, the L13-TPP and the L9-THPP liposomes seem to be sufficiently stable for 7 days when kept at low temperature then could be used in subsequent biological assays.

\subsection{Spray Dryer Liposomes: formulations and characterizations}

As seen, aqueous liposomal dispersions show a considerable chemical and physical instability. This limits the use of liposomes as carriers for biologically active compounds. To solve their limited physical stability, it would therefore be necessary to preserve the compound and the lipids as a dried film, and form the liposomes just before their use, a method that, due to obvious problems of time, space, and poor handling, is not applicable. Consequently, it is interesting to prepare liposomes using a spray dryer [32], i.e. liposomes in a solid state (powder), to be re-suspended in an aqueous vehicle at the time of use, a method also suitable for industrial applications.

In the spray dryer technique, the liposomes are produced in the form of a dried powder starting from a liquid mixture, by rapid drying in the presence of hot air. This is the method of choice for drying many heat sensitive materials, such as food and pharmaceutical compounds. The preparation of liposomes using a spray dryer involves the solubilization of the lipid material in a specific solvent and the drug in the same or different solvent, such as ethanol or methanol, in the presence of antioxidants and water-soluble compounds that act as carriers (maltodextrins, sucrose, PVP). The two phases are kept stirred and then sprayed at a suitable temperature (generally between 50 and $80^{\circ} \mathrm{C}$ ), forming a fine dry particulate of the liposomal components [33].

Three PS-free atomization tests were carried out with different starting mixtures, using a total of $200 \mathrm{~g}$ of liquid phase (solvent/water) (Table 7). Later, for the preparation of dry liposomes charged with a PS, only THPP was used because of its good solubility in ethanol.

Among the three formulations, only the SD3 showed uniform dimensions and visual homogeneity of the powder. Consequently, it was then prepared with the addition of a solution of THPP $(0.006 \mathrm{~g} / 200 \mathrm{~g})$ in ethanol (SD3-THPP). $2.5 \mathrm{~g}$ of the powder obtained from SD3-THPP was re-suspended in 1X PBS buffer, sonicated for $2 \mathrm{~min}$, and characterized. The homogeneity of the product as well as the absence of crystals was confirmed by a microscope analysis. The
Table 7 Composition of the "white" liposomal formulations spray dryer

\begin{tabular}{|c|c|}
\hline & Composition \\
\hline SD1 & $\begin{array}{l}0.335 \% \text { Lipoid S100 + 0.129\% Lipoid DSPG-Na }+0.035 \% \text { Cholesterol }+0.001 \% \\
\alpha \text {-tocopherol }+10 \% \text { Maltodextrin }+89.5 \% \text { solvent/water mixture }\end{array}$ \\
\hline $\mathrm{SD} 2$ & $0.7 \%$ Lipoid S100+0.03\% Cholesterol $+3 \%$ Sorbitol $+96.27 \%$ solvent/water mixture \\
\hline SD3 & $0.7 \%$ Lipoid S100 + $0.03 \%$ Cholesterol $+3 \%$ Sorbitol $+4 \% \mathrm{PVP}+92.27 \%$ solvent $/$ water mixture \\
\hline
\end{tabular}


analysis with Nanophox gave the $151.44 \mathrm{~nm}$ average dimension of SD3-THPP and, as shown in Fig. 6, the formulation is particularly homogeneous.

\subsection{Biological analyses}

The two MEs and the two liposomes showing the greatest stability for each PS (ME1-THPP, E3-TPP, L9-THPP and L13-TPP) were tested in vitro on the SKOV3 cell line to evaluate their photodynamic efficacy. The evaluation of the cytotoxic effect was obtained with MTT assay that allows to determine the $\mathrm{IC}_{50}$ values following exposure to increasing doses of PS under constant light dose. With the same test, it is also possible to determine whether the compounds have intrinsic cytotoxic activity, by administering the same doses of PS, omitting the illumination step [34]. To limit contamination phenomena, previous their use, all the formulations tested were filtered with a $0.2 \mu \mathrm{m}$ syringe filter under a laminar flow hood.

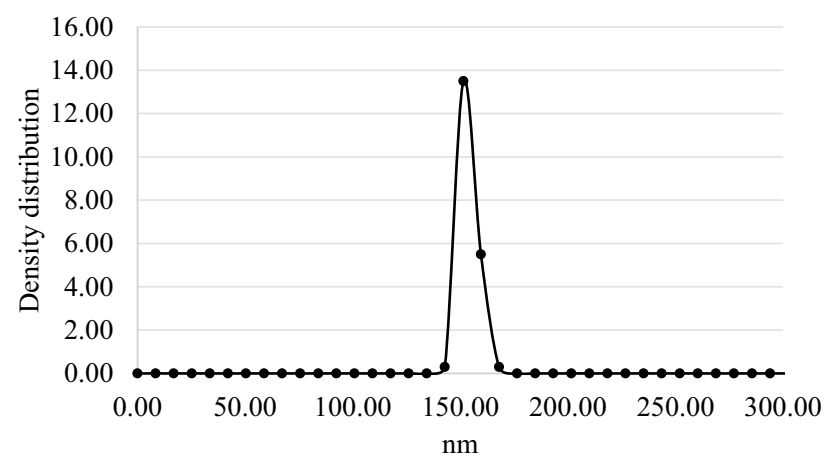

Fig. 6 Analysis concerning the particle size distribution of SD3THPP liposomes performed by Nanophox Sympatec
The toxicity of the "white" formulations, corresponding to the selected ME1-THPP, E3-TPP, L9-THPP and L13-TPP mixtures, was first evaluated to exclude any possible cytotoxic effect of the excipients, either following irradiance or keeping the cells in the dark.

As none of the "white" formulations has shown toxicity, this allows proceeding with the test of the formulations charged with the photosensitizers.

For the four formulations and for THPP in DMSO, the $\mathrm{IC}_{50}$ value was obtained by treating the cells with the solutions diluted in DMEM-F12. After $24 \mathrm{~h}$ of dark incubation, the cells were illuminated for $2 \mathrm{~h}$; after another $24 \mathrm{~h}$ of dark incubation, cell viability was determined by the MTT test (Fig. 7, cell proliferation curves Fig. 5SI). In the case of THPP, the results could be compared with the data obtained with the same molecule formulated in DMSO/DMEM mixture whereas a similar comparison could not be made in the case of TPP because, as stated above, the DMSO/DMEM mixture was found not stable in the time.

The emulsion and the liposomal formulation of TPP allow evaluating the activity of a highly lipophilic molecule that cannot be tested in DMSO/DMEM solution due to its insolubility. The two formulations showed significantly different photodynamic activity. Lovcinsky has reported that the TPP formulation in liposomes did not affect singlet oxygen production [22], and more papers report a similar result in the case of microemulsions [35, 36]. Furthermore, considering the similar stability of the two systems, the data suggest that the greater activity of TPP in the liposomal formulation is attributable to greater cellular penetration.

In a previous work, a similar liposomal formulation of TPP had been tested on HeLa cells showing partial toxicity in the dark and good photodynamic activity, evaluated with tryptophan photodegradation [37]. In this work, no dark toxicity was observed on the SKOV3 cell line while
Fig. $7 \mathrm{IC}_{50}$ values (nM) obtained in SKOV3 cells following treatment with TPP in solvent (n.d.), in emulsion (E3-TPP), and in liposome (L13-TPP) and with THPP in DMSO/DMEM, in microemulsion (ME1-THPP), and in liposome (L9-THPP). Scheduled test time: $24 \mathrm{~h}$ incubation, $2 \mathrm{~h}$ irradiation and $24 \mathrm{~h}$ incubation in drug-free medium, followed by MTT test (mean \pm ES of four independent experiments; ${ }^{*} p<0.05$ L13-TPP vs E3-TPP; $\# p<0.05$, L9-THPP vs THPP; @ $p<0.05$ L9-THPP vs ME1THPP)

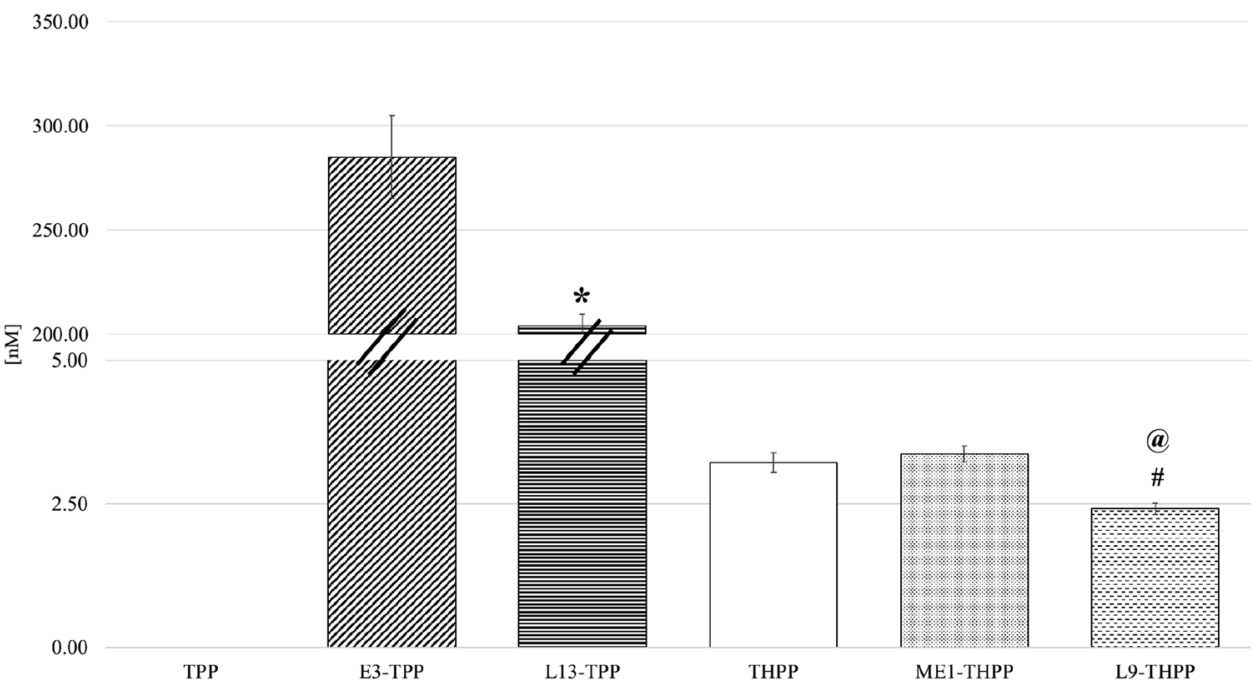


photodynamic activity in the order of $200 \mathrm{nM}$ was observed. It should be emphasized that the result obtained is comparable to the theoretical value predicted by the predictive quantitative structure-activity relationship (QSAR) regression model, already reported by our group [38]. In the case of THPP, the $\mathrm{IC}_{50}$ values obtained with the microemulsion and the porphyrin dispersed in DMSO/DMEM were comparable, confirming the goodness of ME as a delivery system for this type of PS. These data are particularly interesting by considering both the simplicity of the preparation of ME and its stability and biocompatibility.

THPP formulated at the liposomal level has a significant greater photodynamic efficacy compared to both THPP in DMSO/DMEM solution and formulated in ME. This result is in contrast with the results previously reported by Nawalany, which has found that THPP formulated in liposomes exhibits lower photodynamic activity than THPP in DMSO on two tumor cell lines (HCT116 and DU145) [24]. Consequently, it can be assumed that the different composition of liposomes used become decisive in the greater photodynamic efficacy.

The activity of THPP formulated in liposomes prepared with the spray-dryer technique (SD3-THPP) was also evaluated. Preliminary data showed that the photodynamic activity was comparable with L9-THPP (data not shown), this suggests that the preparation technique does not significantly affect the characteristics and the efficacy of the liposome.

The efficiency of PDT treatment is highly dependent on the photosensitizer cellular uptake and accumulation in malignant tissues [39, 40]. Also, intracellular aggregation state of the photodynamic agent is very important, since aggregates are not active in PDT. In this study, the cellular uptake of the THPP entrapped in ME and in liposome was evaluated in SKOV3, comparing the results with those obtained with THPP in DMSO/DMEM solution. To assess the cellular uptake, the cytofluorimetric analysis was used.
The graphs obtained after $24 \mathrm{~h}$ incubation in the presence of $100 \mathrm{nM}$ of the THPP, ME1-THPP and L9-THPP are shown in Fig. 6SI and the related MFI values are reported in Fig. 8. The data show that THPP in DMSO/DMEM or dispersed in the microemulsion (M1-THPP) or in liposome (L9-THPP) was able to accumulate in SKOV3 cells at different levels, compared to control (untreated) cells. The uptake of TPP formulations has not been analyzed as it is not possible to compare the results with TPP as it is not soluble in any solvent that can be used for in vitro tests.

From the results reported in Fig. 8, it is observed that THPP dissolved in solution shows a comparable uptake to THPP in ME, instead it is evident that the liposomal formulation shows a higher uptake than THPP in solution.

The uptake results were in agreement with the photodynamic activity data expressed as $\mathrm{IC}_{50}$ shown in Fig. 7. This may lead to the assumption that the liposomal formulation facilitates PSs cellular penetration and then the killing efficacy. These data seem comparable with that reported by Nawalany who observed how the formulation liposomal showed greater uptake than THPP in HCT116, although the same author observed a lower photodynamic activity attributed to a different cellular localization of the THPP in DMSO or formulated in liposomes [24].

To give further biological information, the cell penetration of the liposomal formulations of the two porphyrins was considered (Fig. 9). The analyses were conducted by treating the cells with a concentration ten times higher than the respective $\mathrm{IC}_{50}$.

The cytoplasmic localization of porphyrins is well known $[41,42]$. The results of the confocal analyses indicate that the different types of carriers do not affect the site of penetration. Actually, the images show the presence of fluorescent spots at the cytoplasmic level, whereas they are absent in the nucleus, thus confirming the effective penetration
Fig. 8 Cellular uptake in SKOV3 cell lines following treatment with THPP, ME1THPP and L9-THPP $24 \mathrm{~h}$ and flow cytometric analysis (mean ES of 3 independent experiments; \#\#p<0.01 vs Ctrl; $* p<0.05$ vs THPP)

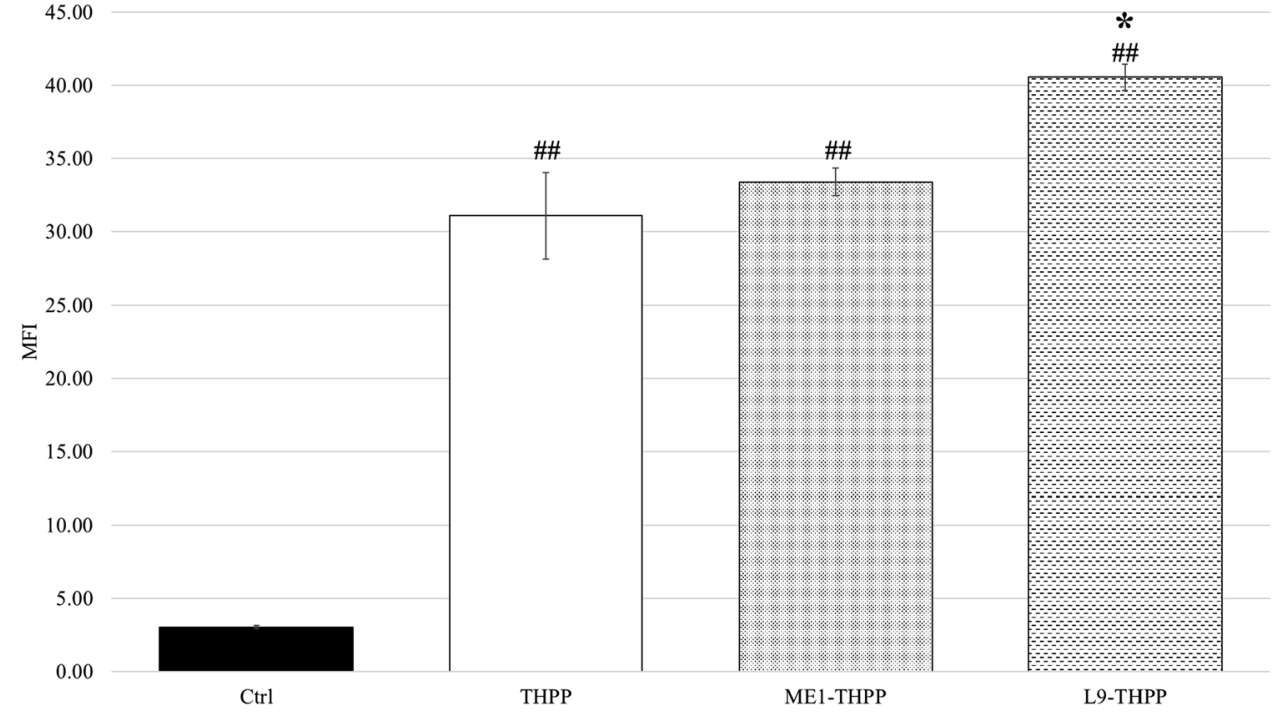


Fig. 9 Confocal microscope images for SKOV3 cells treated with L13-TPP (A) and L9-THPP (B)
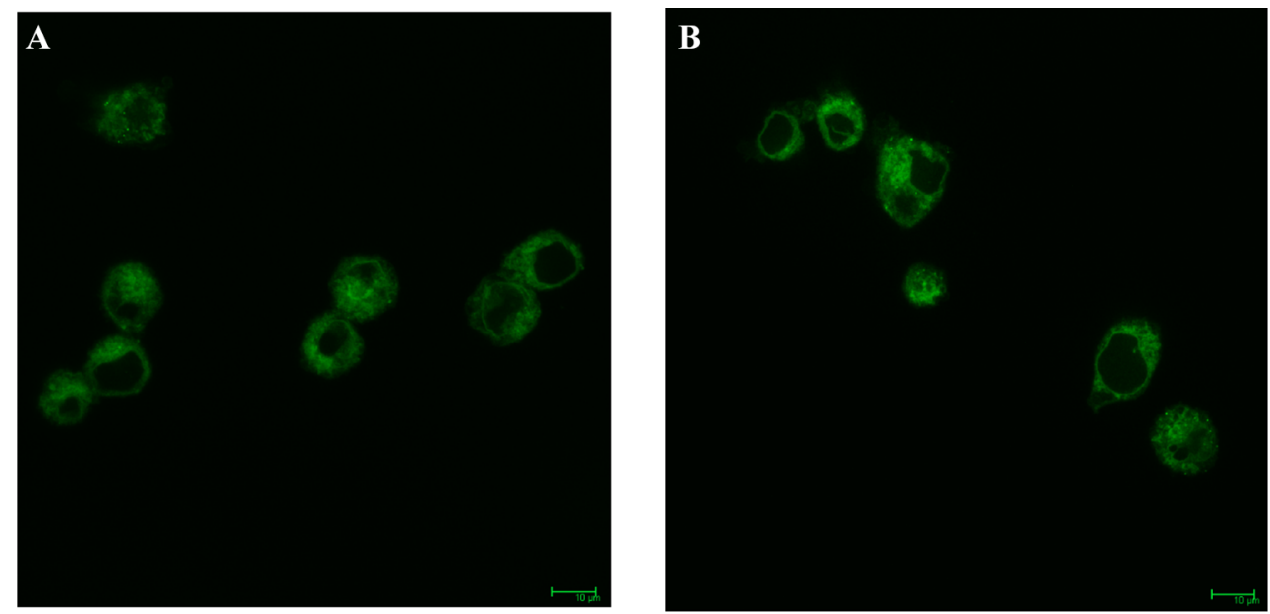

of the porphyrins within cells, even for 5,10,15,20-tetraphenyl porphyrin, the simplest and most lipophilic among the tetraaryl porphyrins, never observed before. This result opens a new frontier for the application in PDT of easily synthesizable photosensitizers, therefore producible in bulk quantity, which can be transformed, by conventional chemical methods, in the corresponding red-adsorbing chlorins, which are suitable for in vivo PDT treatment.

\section{Conclusion}

In the present work, we reported the preparation and photodynamic efficacy of two different types of formulations (ME and liposomes) towards human ovarian adenocarcinoma cell lines. They contain two photosensitizing agents of the porphyrin family, TPP and THPP, both of which are hydrophobic. The two PSs were solubilized in MEs and liposomes which were found stable under the various conditions tested. For both PSs, the liposomal formulation proved to be the most effective for the photodynamic activity of PS, and, in the case of THPP, a higher cell penetration was observed than the porphyrin in DMSO/DMEM solution. The use of liposomes as vehicles carrying hydrophobic photosensitizing molecules has been shown to be advantageous for their use in PDT, actually liposomes allow their efficient transfer and accumulation in cells in non-aggregated form. In conclusion, it appears that the system in which the two porphyrins are incorporated into liposomes may provide a method to increase the selectivity and efficiency of PDT by reducing aggregation, improving tumor penetration and absorption, and increasing photo-toxicity in vitro.

Supplementary Information The online version contains supplementary material available at https://doi.org/10.1007/s43630-021-00076-0.
Funding Open access funding provided by Università degli Studi dell'Insubria within the CRUI-CARE Agreement. This research received no external funding.

\section{Declarations}

Conflict of interest There are no conflicts to declare.

Open Access This article is licensed under a Creative Commons Attribution 4.0 International License, which permits use, sharing, adaptation, distribution and reproduction in any medium or format, as long as you give appropriate credit to the original author(s) and the source, provide a link to the Creative Commons licence, and indicate if changes were made. The images or other third party material in this article are included in the article's Creative Commons licence, unless indicated otherwise in a credit line to the material. If material is not included in the article's Creative Commons licence and your intended use is not permitted by statutory regulation or exceeds the permitted use, you will need to obtain permission directly from the copyright holder. To view a copy of this licence, visit http://creativecommons.org/licenses/by/4.0/.

\section{References}

1. Agostinis, P., Berg, K., Cengel, K. A., Foster, T. H., Girotti, A. W., Gollnick, S. O., Hahn, S. M., Hamblin, M. R., Juzeniene, A., Kessel, D., Korbelik, M., Moan, J., Mroz, P., Nowis, D., Piette, J., Wilson, B. C., \& Golab, J. (2011). Photodynamic therapy of cancer: an update. CA Cancer Journal for Clinicians, 61, 250-281.

2. Monro, S., Colón, K. L., Yin, H., Roque, J., Konda, P., Gujar, S., Thummel, R. P., Lilge, L., Cameron, C. G., \& McFarland, S. A. (2019). Transition metal complexes and photodynamic therapy from a tumor-centered approach: Challenges, opportunities, and highlights from the development of TLD1433. Chemical Reviews, 119, 797-828.

3. Dolmans, D. E., Fukumura, D., \& Jain, R. K. (2003). Photodynamic therapy for cancer. Nature Reviews Cancer, 3, 380-387.

4. Kharkwal, G. B., Sharma, S. K., Huang, Y. Y., Dai, T., \& Hamblin, M. R. (2011). Photodynamic therapy for infections: Clinical applications. Lasers in Surgery and Medicine, 43, 755-767. 
5. Macdonald, I. J., \& Dougharty, T. J. (2001). Basic principles of photodynamic therapy. Journal of Porphyrins and Phthalocyanines, 5, 105-129.

6. Caruso, E., Ferrara, S., Ferruti, P., Manfredi, A., Ranucci, E., \& Orlandi, V. T. (2011). Enhanced photoinduced antibacterial activity of a BODIPY photosensitizer in the presence of polyamidoamines. Lasers in Medical Science., 33(6), 1401-1407.

7. Nawalany, K., Rusin, A., Kepcynski, M., Filipczak, P., Kumorek, M., Kozik, B., Weitman, H., Ehrenberg, B., Krawczyk, Z., \& Nowakowska, M. (2012). Novel nanostructural photosensitizers for photodynamic therapy: In vitro studies. International Journal of Pharmaceutics, 430, 129-140.

8. Mesquita, M. Q., Dias, C. J., Gamelos, S., Fardilha, M., Neves, M. G. P. M. S., \& Faustino, M. A. F. (2018). An insight on the role of photosensitizer nanocarriers for photodynamic therapy. Anais da Academia Brasileira de Ciências, 90(1 Suppl. 2), 1101-1130.

9. Hossen, S., Hossain, M. K., Basher, M. K., Mia, M. N. H., Rahman, M. T., \& Jalal-Uddin, M. (2019). Smart nanocarrier-based drug delivery systems for cancer therapy and toxicity studies: A review. Journal of Advance Research, 15, 1-18.

10. Boyle, R. W., \& Dolphin, D. (1996). Structure and biodistribution relationships of photodynamic sensitizers. Photochemistry and Photobiology, 64, 469-485.

11. Ricchelli, F. (1995). Photophysical properties of porphyrins in biological membranes. Journal of Photochemistry and Photobiology B: Biology, 29, 109-118.

12. Taillefer, J., Jones, M. C., Brasseur, N., van Lier, J. E., \& Leroux, J. C. (2000). Preparation and characterization of $\mathrm{pH}$-responsive polymeric micelles for the delivery of photosensitizing anticancer drugs. Journal of Pharmaceutical Sciences, 89, 52-62.

13. Lucky, S. S., Soo, K. C., \& Zhang, Y. (2015). Nanoparticles in Photodynamic therapy. Chemical Reviews, 115, 1990-2042.

14. Kale, S. N., \& Deore, S. L. (2017). Emulsion micro emulsion and nano emulsion: A review. Systematic Reviews in Pharmacy, 8 , 39-47.

15. Talegaonkar, S., Azeem, A., Ahmad, F. J., Khar, R. K., Pathan, S. A., \& Khan, Z. I. (2008). microemulsion: A novel approach to enhanced drug delivery. Recent Patents on Drug Delivery \& Formulation, 2, 238-257.

16. Tang, J. L., Sun, J., \& He, Z. G. (2007). Self-Emulsifying drug delivery systems: Strategy for improving oral delivery of poorly soluble drugs. Current Drug Therapy, 2, 85-93.

17. Ventola, C. L. (2017). Progress in nanomedicine: Approved and investigational nanodrugs. Pharmacy and Therapeutics, 42, 742-755.

18. Torchilin, V. P. (2005). Recent advances with liposomes as pharmaceutical carriers. Nature Reviews Drug Discovery, 4, 145-160.

19. Mallick, S., \& Choi, J. S. (2014). Liposomes: Versatile and biocompatible nanovescicles for efficient biomolecules delivery. Journal of Nanoscience and Nanotechnology, 14, 755-765.

20. Pegaz, B., Debefve, E., Borle, F., Ballini, J.-P., van der Bergh, H., \& Niamien-Kovakou-Konan, Y. (2005). Encapsulation of porphyrins and chlorins in biodegradable nanoparticles: the effect of dye lipophilicity on the extravasation and the photothrombic activity. A comparative study. Journal of Photochemistry and Photobiology B: Biology, 80, 19-27.

21. Shan, J., Budijono, S. J., Hu, G., Yao, N., Kang, Y., Ju, Y., \& Prud'homme, R. K. (2011). Pegylated composite nanoparticles containing upconverting phosphors and meso-tetraphenyl porphine (TPP) for photodynamic therapy. Advanced Functional Materials., 21, 2488-2495.

22. Lovcinsky, M., Borecky, J., Kubat, P., \& Jezek, P. (1999). Mesotetraphenylporphyrin in liposomes as suitable photosensitizer for photodynamic therapy of tumors. General Physiology and Biophysics, 18, 107-118.
23. Desroches, M.-C., Kasselouri, A., Meyniel, M., Fontaine, P., Goldmann, M., Prognon, P., Maillard, P., \& Rosilio, V. (2004). Incorporation of glycoconjugated porphyrin derivatives into phospholipid monolayers: a screening method for the evaluation of their interaction with a cell membrane. Langmuir, 20, 11698-11705.

24. Nawalany, K., Rusin, A., Kepczynski, M., Mikhailov, A., KramerMarek, G., Snietura, M., Poltowicz, J., Krawczyk, Z., \& Nowakowska, M. (2009). Comparison of photodynamic efficacy of tetraarylporphyrin pegylated or encapsulated in liposomes: In vitro studies. Journal of Photochemistry and Photobiology B: Biology, 97, 8-17.

25. Yumoto, T., Satake, S., Hino, S., Sugikawa, K., Kawasaki, R., \& Ikeda, A. (2020). Improved water solubility and photodynamic activity of hydroxy-modified porphyrins by complexation with cyclodextrin. Organic \& Biomolecular Chemistry, 18, 6702-6709.

26. Rickwood, D. (1990). In: Liposomes: a practical approach vol 3, Ed. R. R. C. New, Oxford University Press, pp 105-160.

27. Caruso, E., Gariboldi, M., Sangion, A., Gramatica, P., \& Banfi, S. (2017). Synthesis, photodynamic activity, and quantitative structure-activity relationship modelling of a series of BODIPYs. Journal of Photochemistry and Photobiology B: Biology, 167, 269-281.

28. Caruso, E., Cerbara, M., Malacarne, M. C., Marras, E., Monti, E., \& Gariboldi, M. B. (2019). Cationic diarylporphyrins: In vitro versatile anticancer and antibacterial photosensitizers. Journal of Photochemistry and Photobiology B: Biology, 17, 111548.

29. Malacarne, M. C., Banfi, S., \& Caruso, E. (2020). In vitro photodynamic treatment of cancer cells induced by aza-BODIPY. Photochemical \& Photobiological Sciences, 19, 790-799.

30. Zhang, H. (2017). In: Liposomes, vol 2. Ed. G. G. M. D'Souza, Humana Press, 2nd edition, pp 17-22.

31. Brandl, M. (2001). Liposomes as drug carriers: A technological approach. Biotechnology Annual Review., 7, 59-85.

32. Goldbach, P., Brochart, H., \& Stamm, A. (1993). Spray-drying of liposomes for a pulmonary administration. Drug Development and Industrial Pharmacy, 19, 2623-2636.

33. Patel, R. P., Patel, M. P., \& Suthar, A. M. (2009). Spray drying technology: An overview. Indian Journal of Science and Technology, 2, 44-47.

34. Caruso, E., Malacarne, M. C., Banfi, S., Gariboldi, M. B., \& Orlandi, V. T. (2019). Cationic diarylporphyrins: in vitro versatile anticancer and antibacterial photosensitizers. Journal of Photochemistry and Photobiology B: Biology, 197, 111548.

35. Quiroz-Segoviano, R. I. Y., García-Sánchez, M. A., DelgadoBuenrostro, N. L., Chirino, Y. I., Bernal-Chávez, S., NavaArzaluz, M. G., \& Ganem-Rondero, A. (2019). Tetraphenylporphyrin intended for use in photodynamic therapy: Influenceof sonophoresis and the formulation (solution or microemulsion) onpercutaneous penetration. Journal of Drug Delivery Science and Technology., 53, 101145.

36. Wang, S., Gao, R., Zhou, F., \& Selke, M. (2004). Nanomaterials and singlet oxygen photosensitizers: Potential applications in photodynamic therapy. Journal of Materials Chemistry, 14, 487-493.

37. Canete, M., Villanueva, A., Dominguez, V., Polo, S., Juarranz, A., \& Stockert, J. C. (1998). Meso-tetraphenylporphyrin: Photosensitizing properties and cytotoxic effects on cultured tumor cells. International Journal of Oncology, 13, 497-504.

38. Banfi, S., Caruso, E., Buccafurni, L., Murano, R., Monti, E., Gariboldi, M., Papa, E., \& Gramatica, P. (2006). Comparison between 5,10,15,20-Tetraaryl- and 5,15-Diarylporphyrins as photosentitizers: Synthesis, photodynamic activity, and quantitative structureactivity relationship modeling. Journal of Medicinal Chemistry, 49, 3293-3304. 
39. Ochsner, M. (1997). Photophysical and photobiological processes in the photodynamic therapy of tumors. Journal of Photochemistry and Photobiology B: Biology, 39, 1-18.

40. Weizman, E., Rothmann, C., Greenbaum, L., Shainberg, A., Adamek, M., Ehrenberg, B., \& Malik, Z. (2000). Mitochondrial localization and photodamages during photodynamic therapy with tetraphenylporphyrines. Journal of Photochemistry and Photobiology B: Biology, 59, 92-102.
41. Osterloh, J., \& Vicente, M. G. H. (2002). Mechanisms of porphyrinoid localization in tumors. Journal of Porphyrins and Phthalocyanines, 06, 305-324.

42. Banfi, S., Caruso, E., Caprioli, S., Mazzagatti, L., Canti, G., Ravizza, R., Gariboldi, M. B., \& Monti, E. (2004). Photodynamic effects of porphyrin and chlorin photosensitizers in human colon adenocarcinoma cells. Bioorganic \& Medicinal Chemistry., 12, $4853-4860$ 